\title{
Oscillatory priming and form complexity
}

\author{
ZhUANGHUA SHI \\ Ludwig-Maximilians-Universität München, Munich, Germany \\ AND \\ MARK A. ELLIOTT \\ National University of Ireland, Galway, Ireland
}

\begin{abstract}
Detection of a target grouping is expedited when the target display is preceded by presentation of an oscillating premask that includes priming elements presented intraphasically and below detection threshold at the display locations subsequently occupied by the target. Five experiments were performed to investigate how priming is affected by both the complexity and the geometry of prime/target forms. Experiment 1 showed that the subjective complexity of different polygons was coded in accord with an objective measure of form complexity. Experiments 2 and 3 revealed oscillatory priming to increase as a function of the subjective complexity of prime/target forms when those forms were regular and predictable. However, Experiments 4 and 5 showed that this relation did not hold when the prime/target forms were irregular and unpredictable. Taken together, it is argued that both subjective complexity and the Prägnanz quality of the prime/target forms come to determine the magnitude of priming. These results are discussed with reference to current physiological hypotheses regarding perceptual organization.
\end{abstract}

The Gestalt psychologists (Koffka, 1922; Köhler, 1947; Wertheimer, 1912) proposed that perceptual organization is subject to a number of organizing principles, including proximity, good continuation, similarity, and closure. In addition, items group according to dynamic principles, such as common fate and prior experience. In his famous law of Prägnanz, Wertheimer stated that the phenomenal organization of a percept will be as "good" as the prevailing conditions allow. Koffka (1935) proposed that the law of Prägnanz can be identified with a so-called minimum principle, where goodness, or Prägnanz quality, is identified with the relative simplicity of a given perceptual organization. According to the minimum principle, the perceptual system seeks to reduce its engagement to a minimum and, thus, to describe the external world in the simplest possible way. Gestalt psychology thus held that the modus operandi of perceptual organization is essentially dynamic in character, which is seemingly consistent with a natural tendency of physical systems to settle into minimum energy states and is at least thematically consistent with recent theories concerning the dynamic characteristics of brain activity that arise during perception.

Of particular relevance to the idea that the dynamics of brain activity are responsible for perceptual organization are the results of a number of physiological studies of the neuronal response to the grouping of visual stimuli. The outcome of these studies is taken to indicate that neuronal synchronization brings about the binding together of different visual elements (see Eckhorn et al., 1988; Engel,
Kreiter, König, \& Singer, 1991; Gray, König, Engel, \& Singer, 1989; Livingstone, 1996). Specifically, synchronized oscillatory activity arises within the striate cortex and between the striate and the extrastriate cortex in response to the presence of simple line segments in grouping situations - for example, moving bars that share a common trajectory (i.e., common fate motion; Engel et al., 1991; Gray et al., 1989; Livingstone, 1996). In addition, for visual field separations of less than $5^{\circ}$, under which conditions stimuli may be considered to be proximal, synchrony has been observed between striate cortical neurons (Ts'o \& Gilbert, 1988; Ts'o, Gilbert, \& Wiesel, 1986), whereas a strong tendency for synchronous firing between striate cortical neurons with similar orientation preferences has been considered a possible neural correlate with the coding of stimulus similarity (Eckhorn et al., 1988). The studies of Gray et al., in addition to that of Freiwald et al., have also provided some evidence that contour collinearity promotes synchronization between striate cortical neurons (Freiwald, Kreiter, \& Singer, 1995; Gray et al., 1989). Finally, and with respects to the coding of neighboring objects (such as figure vs. ground), the modulation frequencies and phases that characterize the response to figure and ground have been shown to be statistically independent, suggesting that figure-ground segmentation may be subserved by a form of temporal segregation of neural codes for figure and ground (see Castelo-Branco, Goebel, Neuenschwander, \& Singer, 2000; Gail, Brinksmeyer, \& Eckhorn, 2000). Given that neuronal synchrony accompanies the coding of sepa- 
rate relations between different visual sets, it seems plausible to conceive of neuronal synchronization as a potential method for organizing the visual environment into its simplest components. In other words, neuronal synchronization may be a neuronal correlate of the minimum principle. In order to test this hypothesis, we examined the effects of stimulus phase synchronization upon the coding of spatial organizations that formed shapes of different complexities, under the following assumptions.

First, it was assumed that measures of stimulus phase synchronization are analogous to those of neuronal synchronization. This assumption was made, in the first case, on the basis of evidence that stimuli will respond at the rhythm of an external driving stimulus; for example, visuocortical neurons can adopt the rhythm of flickering stimulus presentation (Rager \& Singer, 1998), with perceptual consequences related to the processing of form (see Gur, Beylin, \& Snodderly, 1997). Other evidence in support of this idea will be reviewed in consideration of the paradigm used in this study and will be detailed in later paragraphs. Second, as was mentioned above, evidence for spatial organization based on the minimum principle has shown that perceptual grouping depends on relational factors, such as stimulus symmetry, regularity, set, good continuation, and so forth. Stimulus configurality may vary along one or more of a number of dimensions of complexity. These have been the subject of both introspective studies and formal modeling undertaken by several research groups in the past half century (Arnoult, 1960; Chen \& Chen, 1982, 1987; Garner, 1970, 1974; Leeuwenberg, 1968; Mavrides \& Brown, 1969; Palmer, 1991; for a review, see Donderi, 2006). From these studies, a number of factors have been found to influence the perceived complexity of simple 2-D forms. Arnoult, for example, showed that the subjective complexity of 2-D forms was related to the number of independent sides, the ratio of perimeter ${ }^{2} /$ area, and form symmetry. On the basis of the observation that good forms have fewer transformational variants, Garner proposed a transformational approach to account for the perceived complexity of patterns. According to this theory, the perceived complexity of a given visual pattern relates implicitly to a set of alternative patterns (referred to as rotation and reflection subsets). The more complex the pattern, the greater the number of subsets is. In a modification to Garner's approach, Palmer introduced the concept of plane isometry, which distinguished a finer grading of pattern complexity: For example, a pattern with mirror symmetry was judged to be less complex than one with $180^{\circ}$ rotational symmetry, although in Garner's approach, both would have the same subset size (Palmer, 1991).

These studies offer a number of measures of perceived complexity that are essentially reducible to the pattern of relational codes describing the organization of visual features. Complexity is intrinsically related to the operation of the minimum principle. The question of whether or not measures of complexity may be thus considered to correlate with some measure of neuronal binding (e.g., the effectiveness of neuronal synchronization) remains to be seen. Even so, the obvious relationship between the two levels of explanation is strongly suggestive of the possibil- ity that perceived complexity describes something of the efficiency or inefficiency of the binding process, whereas variations in binding may be a measure of variation in processes obeying the minimum principle.

Our third assumption concerns the fact that at least some types of visual relations are coded very early in perceptual processing and may not be open to introspective analysis (Neisser, 1967). This conclusion seems consistent with the physiological studies noted above, at least if one takes anatomy as the main criterion: Most of the aforementioned recordings of neuronal synchrony were taken from primary visual areas, at which stage the immediate outcomes of visual processing are not available for consciousness (see, e.g., Crick \& Koch, 1995, 1998; He, Cavanagh, \& Intriligator, 1996). Given that the processes of interest lie below conscious thresholds, the direct effects of stimulus phase synchronization on perceptual organization (Kandil \& Fahle, 2001; Lee \& Blake, 1999) may be difficult to evaluate. For example, directly comparing temporal asynchrony and spatial displacement has led to the uncertain conclusion that temporal synchrony is of little influence for the perception of spatial organization (Fahle \& Koch, 1995). On this basis, a perceptually implicit measure may be an appropriate method for evaluating the idea that variations in the effects of stimulus phase synchronization relate to variations in the complexity of stimulus forms.

Perceptually implicit measures of binding, which are, at the same time, a function of stimulus phase synchronization, have been described by Usher and Donnelly (1998), Parton, Donnelly, and Usher (2001), and Elliott and Müller $(1998,2000,2001)$. In each of these cases, two or more image frames were rapidly and repeatedly presented, so that the combination of high-frequency presentation with close to 0 -msec interframe intervals rendered the content structure of a given image frame nondeterminable. Despite this, Usher and Donnelly found a bias toward reportage consistent with the orientation of groupings presented in one of two indistinguishable image frames. In Elliott and Müller's experiments, a priming stimulus (comprising four crosses presented in square arrangement) occupied one of four separate image frames that, when combined, formed a regular $3 \times 3$ element premask matrix (Figure 1). Image frames (comprising the priming and the other premask matrix crosses) were presented one after another at a frequency of $10 \mathrm{~Hz}$, with a frame onset asynchrony of $25 \mathrm{msec}$ (equivalent to a frame-by-frame presentation frequency of $40 \mathrm{~Hz}$ ) and with an interframe interval of less than $1 \mathrm{msec}$. In this way, the priming crosses were presented in a regular spatial pattern and, at the same time, synchronously or intraphasically. The combination of high-frequency presentation with $<1$-msec interframe interval rendered the content structure of a given image frame nondeterminable (Elliott \& Müller, 1998, Experiment 2). Instead, the phenomenological effect of this temporal modulation was of a stochastic surface flicker across an otherwise static $3 \times 3$ matrix of premask crosses. In Elliott and Müller's task, reaction time (RT) priming effects were recorded to the presence or absence of a Kanizsatype grouping included within a subsequently presented 


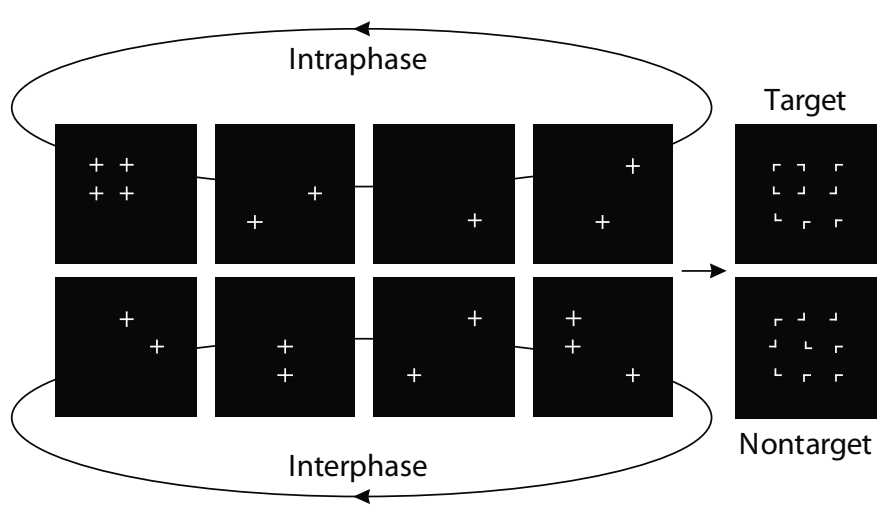

Figure 1. Schematic illustration of Elliott and Müller's (1998, 2000, 2001) paradigm. The left-hand side of the figure shows example sequences of the four premask subset frames in the intraphase and interphase conditions. The premask sequence was continually recycled during the presentation time to target display onset, which produces the effect of a flickering matrix, within which it is not possible to determine the composition of the individual frames. The right-hand side of the figure shows examples of target-present and -absent displays.

distractor matrix, the elements of which were presented at the same matrix location as that previously occupied by the repeating priming crosses (that the priming effects were spatially specific was reported by Elliott \& Müller, 1998, Experiment 3). Priming effects, which are also target specific (i.e., they are absent on target-absent trials), were measured in terms of the difference between RTs to targets following intraphasic premask presentation and RTs following interphasic premask presentation (Figure 1). During intraphasic premask presentation, one of four image frames contained four crosses presented at the same matrix locations as the target elements. In the interphasic premask condition, all the crosses, including the four crosses presented at the target locations, were divided pseudorandomly across two or more frames. Mean priming effects commonly vary across the 25 - to $30-\mathrm{msec}$ range and do not vary substantively with variations in the target RTs (Elliott \& Müller, 1998, 2000, 2001).

For presentation at $40 \mathrm{~Hz}$, the intraphase priming crosses were found to generate a prime with close to a $40-\mathrm{Hz}$ structure (Elliott \& Müller, 2000). This may be explained if one considers local prime activity to have inherited the global frequency of premask matrix presentation. Given that the spatial structure of the priming stimulus was nondeterminable and given that the priming stimulus did not encourage the deployment of focal-attentional mechanisms or eye movements to its matrix location, ${ }^{1}$ priming was suggested to be active principally in early visual mechanisms, even though neurons in areas such as the primary visual cortex do not possess receptive fields with sufficient dimensions to be able to respond to the entire premask matrix, the only stimulus presented at a frequency of $\sim 40 \mathrm{~Hz}$. However, later cortical mechanisms do, and on this basis, it was proposed that an early neural prime might become active at $\sim 40 \mathrm{~Hz}$ by virtue of this frequency's being fed back from later mechanisms coding the entire premask matrix and, thus, responding to a $40-\mathrm{Hz}$ staccato of premask-frame-induced activity within their retinally specified receptive fields. A feedforward- feedback model of priming was also suggested from an independent-components decomposition of the EEG accompanying premask matrix presentation. This analysis revealed that the only component describing EEG activity during priming stimulus presentation consisted of coactive sources under posterior and frontal electrodes, with spectral power in the component peaking at $10 \mathrm{~Hz}$ and at around $34 \mathrm{~Hz}$ (Conci, Elliott, Müller, Wendt, \& Becker, 2004; also described in Elliott, Conci, \& Müller, 2003). Although the paradigm of Elliott and Müller offers a basis for analysis of the dynamic system underlying figural prime formation, the data thus far refer to primes and targets in square arrangement and, as a consequence, offer no indication of potential interactions either of stimulus form or complexity or of the effects of more than one possible target upon the efficiency of oscillatory priming. Recent evidence has shown that the primes do include information about the spatial relations between the premask crosses, evidence which clearly suggests the prime to be a form of Gestalt-related grouping (Elliott, Giersch, \& Seifert, 2006). With this in mind, the main goals of the research presented here were to explore whether or not variations in priming performance might be explained by means of variations in figural complexity and, on this basis, whether evidence exists to support the idea of neuronal synchronization as one measure of the minimum principle. In order to derive measures of oscillatory priming as a function of target complexity, we used a number of regular and irregular polygonal forms as our stimuli. In a first step, in Experiment 1, a measure of Prägnant quality of different polygons was taken with the aim of deriving a model of subjective complexity against which data in the four subsequent priming experiments could be compared. In Experiment 2, three regular prime-target forms (triangle, square, and hexagon) were used, and the magnitude of oscillatory priming increased as a function of increasing form complexity. In Experiment 3, a mixture matrix was employed, so that four regular forms (triangle, square/ diamond, pentagon, and hexagon) could be embedded in 

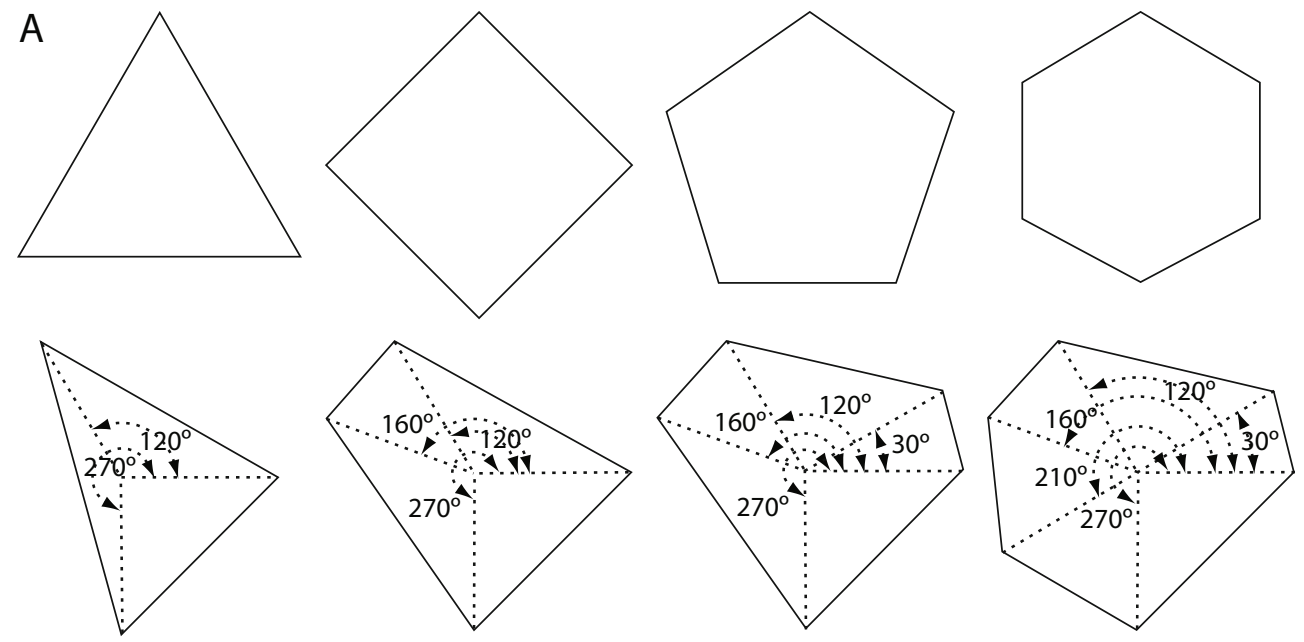

B

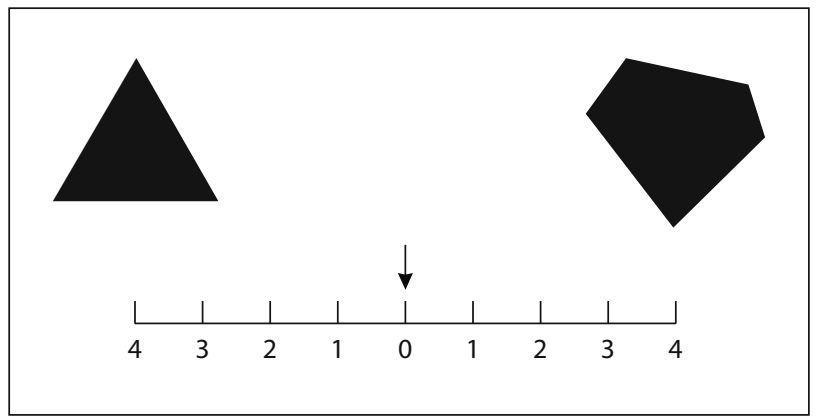

Figure 2. (A) Four regular forms and four irregular forms used for complexity comparisons. The polar angles of the apexes of irregular polygons are specified in the figure. (B) A schematic illustration of the display for the subjective complexity comparison rating procedure.

the same matrix. As with Experiment 2, priming increased with increasing form complexity. In Experiments 4 and 5, four irregular forms (corresponding to irregular three-, four-, five-, and six-sided polygons) were tested in a mixture matrix, and in contrast to the results of Experiments 2 and 3, priming effects remained constant, irrespective of variations in form complexity. These results indicate that oscillatory priming is sensitive to Prägnant quality when the prime/target forms are regular. Conversely, when prime/ target complexity is irregular, it may require additional processing resources, with redundant variations in complexity ignored for the purposes of prime formation.

\section{EXPERIMENT 1}

In Experiment 1, we explored the subjective complexity of a number of different geometrical forms. The main purpose of this experiment was to establish a model of subjective complexity with objective Prägnanz quality for 2-D polygons. The aim of Experiment 1 was to provide data with which the data in the four subsequent priming experiments could be compared.

\section{Method}

Participants. Thirteen participants, all with normal or correctedto-normal vision, participated in Experiment 1 ( 7 of them female; mean age, 30.2 years). The participants were paid at a rate of $€ 8 / \mathrm{h}$.
Apparatus and Stimuli. The stimuli were presented on a 21-in. Sony Trinitron monitor with vertical refresh set to $140 \mathrm{~Hz}$ and with stimulus presentation controlled by Cambridge Research Systems VSG $2 / 3$ graphics card with $16-\mathrm{MB}$ framestore memory. The stimuli were eight 2-D closed-contour forms (illustrated in Figure 2), four of which were regular forms (triangle, square, pentagon, and hexagon), whereas the remaining four were irregular polygons transformed from the regular forms above. The irregular polygons had polar angles as shown in Figure 2. Each form subtended the same visual angle of $2^{\circ} 10^{\prime}$ at a viewing distance of $100 \mathrm{~cm}$ and was $2,400 \mathrm{~cd} / \mathrm{m}^{2}$ on a background field of $6.0 \mathrm{~cd} / \mathrm{m}^{2}$.

Design and Procedure. Prior to the experiment, the participants were given the following heuristic definition of complexity: "A form is complex if it would take more time to recall and is harder to reproduce, and it is simple if it would take less time to recall and is easier to reproduce than the accompanying form." Following a practice block, the participants completed eight experimental blocks of 64 trials each. On each trial, two forms were presented on the left and right of the monitor screen. Beneath these forms, a slider was presented marked from center to both left and right with a scale from 0 to 4 (shown in Figure 2B). The participants were asked to compare the two forms and to indicate (1) which of the two was the more complex and (2) to what extent this form was more complex than the accompanying form. The participants gave both measures of complexity by moving the slider in the direction of the more complex form and to the number (1-4) corresponding to their judgment of how much more complex this form was relative to the other. For matching forms, the participants were asked to choose the central zero scale value even when they had different rotations. The stimuli were displayed until the judgment had been confirmed by a mouse click. 
A paired-comparisons design was employed to derive a measure of form complexity. In this design, 64 complete comparison pairs were presented in each block and constituted a full multifactorial variation of each of the eight forms used in the experiment presented to the right compared with each of the same eight forms to the left of the monitor screen. Both regular and irregular forms were presented at rotations of $0^{\circ}, 90^{\circ}, 180^{\circ}$, and $270^{\circ}$, relative to the horizon. The rotations were considered as a random factor for the purpose of analysis and were balanced over the whole experiment.

\section{Results and Discussion}

In each trial, the judgment value was assigned to the more complex form by the winner-take-all method. Thus, the values could be regarded as interval data that measure the complexity difference from other forms. Those data were first submitted to a three-way within-subjects ANOVA involving the factors of number of sides/apexes, regularity, and rotation. The results showed significant main effects of number of sides/apexes and regularity $[F(3,36)=31.5, p<.01$, and $F(1,12)=16.8, p<.01$, respectively], whereas the main effect of rotation was nonsignificant $[F(3,36)=0.16$, n.s. $]$. On the basis of this, the data were collapsed across rotations and were analyzed only with respect to the number of sides (or apexes) and regularity. A two-way repeated measures ANOVA showed that the main effects of both regularity and number of sides were significant $[F(1,12)=16.9, p<.01$, and $F(3,36)=$ $34.3, p<.01$, respectively]. The two-way interaction between number of sides and regularity was also significant $[F(3,36)=10.6, p<.01]$. Subsequent Tukey tests for multiple comparisons $(p<.05)$ were conducted for the regular and the irregular forms separately. The results of these analyses revealed that the pentagons were the most complex among the regular forms (mean subjective complexity was $1.42 \pm 0.11$, as compared with the hexagon

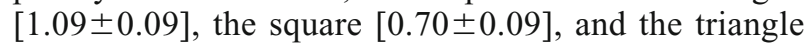
$[0.55 \pm 0.12]$. Among the irregular forms, complexity could be divided into two groups: the six- and five-sided forms versus the quadrilaterals and triangles (the mean subjective complexities were $2.22 \pm 0.19$ for the six-sided and $2.25 \pm 0.20$ for the five-sided forms, as compared with $1.49 \pm 0.15$ for the quadrilaterals and $1.11 \pm 0.17$ for the triangles). These results are summarized in Figure 3A.

For regular forms, subjective complexity achieved its maximum for pentagons, suggesting that the number of sides is not the only factor influencing perceived complexity. Recall that the symmetry properties proposed by both Garner $(1970,1974)$ and Palmer (1991) were also a critical aspect of figural complexity. According to Garner, under certain rotation and mirror transforms, a complex pattern will generate more different forms than will the simpler pattern. For example, with general four-axes mirror reflection and $90^{\circ}, 180^{\circ}$, and $270^{\circ}$ rotation, the possible number of different forms for the irregular triangle was eight, whereas it was only four for the regular triangle. Under the transforms above, there were four different levels of subset sizes (by the method of exhaustion, it is easy to show that the four possible subset sizes were one, two, four, and eight), which reflected four different levels of complexity. Using these four different levels as an index of the symmetry property of the form, we conducted a further analysis of the rela-
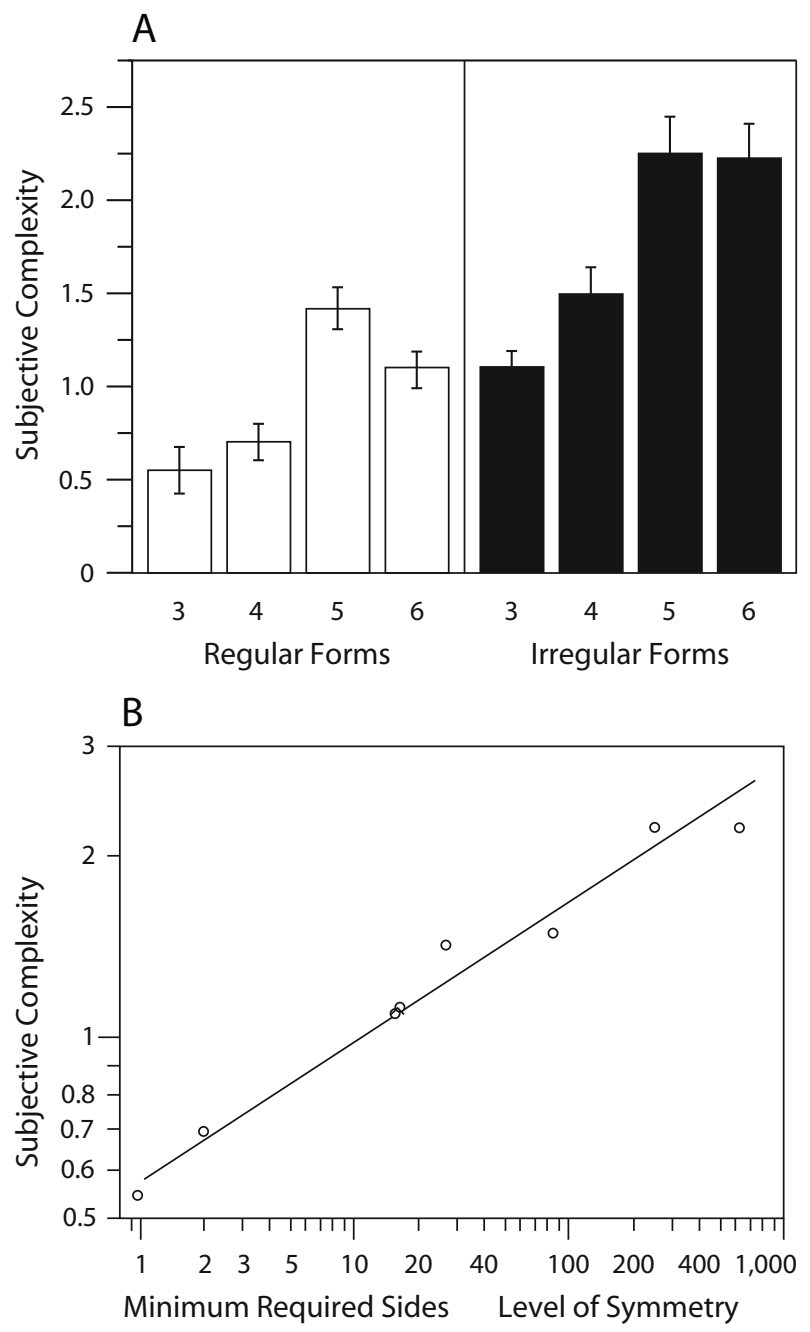

Figure 3. (A) Mean subjective complexity ratings for eight different forms with regular and irregular as subgroups. (B) Loglog plot of subjective complexity against minimum required sides with symmetry level power component. The circle symbols represent subjective complexity.

tionship between subjective complexity, symmetry, and the number of sides or apexes possessed by the form. Consistent with the notion of minimum complexity, we used here the minimum required number of sides for the definition of an $n$-sided form, instead of the total number of sides. Thus, for $n$-sided irregular forms, this requires $n-1$ fixed sides to specify the form, whereas for $n$-sided regular forms, it requires only $n-2$ fixed sides to specify the form.

This analysis revealed that subjective complexity exhibits a power law relation with symmetry level and the number of minimum required sides $\left(r^{2}=.97, p<.01\right.$; see also Figure 3B):

$$
C=0.59 \cdot M^{0.22 \cdot L},
$$

where $C$ denotes subjective complexity, $L$ denotes the level of symmetry (ranging from 1 to 4 ), and $M$ denotes the minimum required sides.

The power law relation above showed that subjective complexity of form was related to minimum required 
A
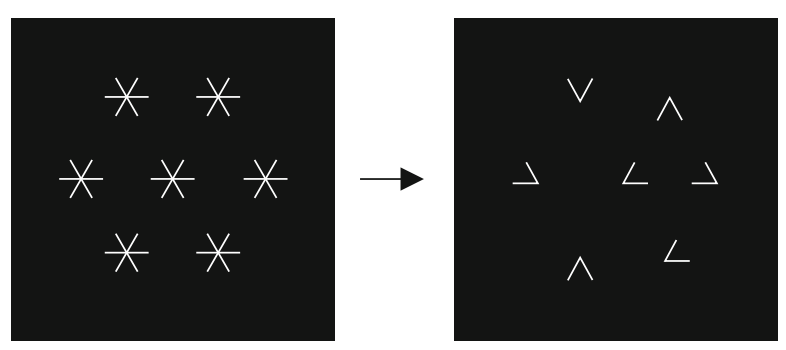

B
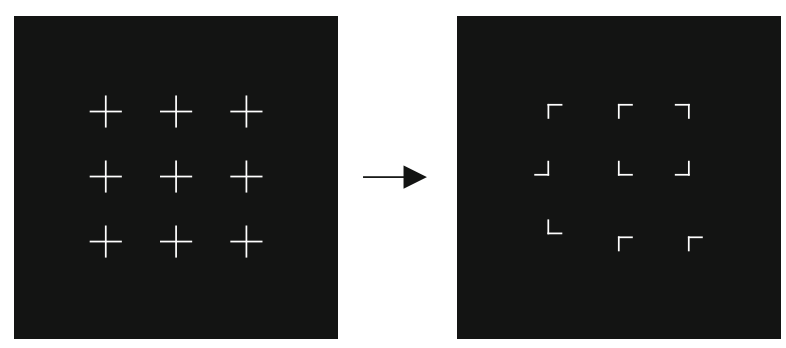

C
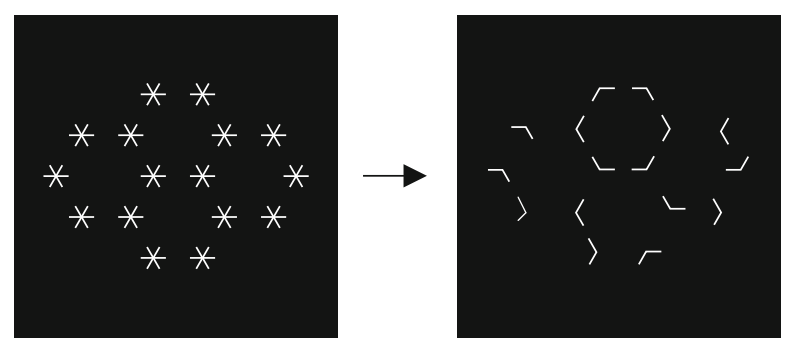

Figure 4. (A) Example of premask and target matrices for triangle prime/targets. (B) Example of flickering display and target display for square matrices. (C) Example of flickering display and target display for hexagon matrices.

sides, which is consistent with the minimum coding idea proposed by Leeuwenberg and van der Helm (Leeuwenberg, 1968; van der Helm \& Leeuwenberg, 1991). The power exponent is related to the level of symmetry, suggesting that the level of symmetry acts as the basis for classification of the different complexity of the forms. In summary, the results of Experiment 1 showed that subjective complexity was related not only to the number of sides, but also to the potential symmetries of the form. Subjective complexity exhibits a power law relation with minimum required sides and symmetry level.

\section{EXPERIMENT 2}

Experiment 2 was performed to explore the effects of potential prime and target complexity on oscillatory priming and was similar to the experiments previously conducted by Elliott and colleagues (Elliott \& Müller, 1998, 2000). Experiment 2 differed in terms of matrix design, and matrices were designed to accommodate a number of prime/target forms. Specifically, three different types of premask/target matrices were used: those supporting triangular, square, and hexagonal prime/target configurations (illustrated in Figure 4). The square matrix display was of the same physical dimensions as those used in previous studies. In this experiment, we omitted the pentagon form, since it afforded no tight matrix structure.

\section{Method}

Participants. Fourteen participants, all with normal or correctedto-normal vision, participated in Experiment 2 ( 9 of them female; mean age, 26.2 years). The participants performed one block of practice trials immediately before the experiment proper and were naive as to the precise experimental conditions presented in the experiment. The participants were paid at a rate of $€ 8 / \mathrm{h}$.

Apparatus and Stimuli. Event timing, data collection, and stimulus frame generation were controlled by an IBM-compatible PC, which also controlled oscilloscopic image presentation by means of a Cambridge Research Systems D300 plotter buffer with 16-MB framestore memory. The stimuli were presented on Datacheck Corporation $1050 \mathrm{XY}$ plotter equipped with a very fast $\mathrm{P} 15$ phosphor. This display technology ensured that image frames were plotted with a potential for temporal control of $500 \mathrm{~Hz}$, and the P15 phosphor ensured that on-screen image persistence was reduced to $10 \%$ of normal image intensity within $2.8 \mu \mathrm{sec}$ of image termination (Bell, 1970).

The stimulus display consisted of a matrix of premask elements distributed across four sequentially presented image frames (illustrated in Figure 1). The sequence of premask image frames was presented for $600 \mathrm{msec}$, after which the premask matrix transformed into a static target matrix of corner junctions (illustrated in Figure 4). Under target conditions, a subset of these junctions would group in one display region to form an illusory target figure. The frequency of premask presentation was decided upon on the basis of previous work that identified priming to be quite specific to premask matrix presentation at $40 \mathrm{~Hz}$ (see, e.g., Elliott \& Müller, 1998). This was achieved by presenting the four individual premask image frames at a rate of 10 repeats/sec, which, given a constant exposure duration of $25 \mathrm{msec}$ and an interframe interval of less than $1 \mathrm{msec}$, resulted in a matrix frequency of $40 \mathrm{~Hz}$. Premask matrix presentation was divided into two critical conditions. In the first condition, the premask elements were pseudorandomly (or interphasically) distributed across all four image frames and were controlled to avoid the possibility of accidental, figurally relevant spatial organizations arising within an image frame. In the second condition, the premask matrix included the synchronous (or intraphase) presentation of a figurally relevant image frame comprising 3, 4, or 6 premask elements in either triangular, square, or hexagonal arrangement. Target matrix presentation contained targetpresent and -absent conditions. On target-present trials three, four, or six corner junctions were grouped to form one of three types of Kanizsa-type target forms (i.e., triangle, square, or hexagon). (Note that Kanizsa-type refers here to the illusory geometrical forms that emerge as a function of the collinear organization of appropriately oriented corner junctions; these are illustrated in Figures 4A, 4B, and 4C.) The location of the target form was identical to the location of the intraphasic premask. On target-absent trials, the target matrix did not include elements that grouped to form an illusory form. An example of the different intraphase and interphase premask element organizations is illustrated in Figure 1. A pilot signal detection experiment showed that the participants (12 participants, 5 of them female; mean age, 26.5 years; all with normal or corrected-to-normal vision; each produced 504 rated judgments for all the conditions) were unable to discern the intraphasic or interphasic structure of trial $\left(A_{z}\right.$ varied from 0.471 to $0.547 ; M=0.508, S E=0.007){ }^{2}$

The experiment was designed to investigate the effects of variations in premask/target geometry upon oscillatory priming and, accordingly, three different matrix patterns consisting of 7, 9, and 16 premask/target elements were constructed to accommodate the three different premask/target forms (illustrated in Figure 4). Unlike either square or hexagon matrices, the matrix used in the triangle condition allowed for six possible prime/target locations, of which 
Table 1

Detailed Configuration of the Stimuli in Experiment 2

\begin{tabular}{|c|c|c|c|c|c|}
\hline \multirow[b]{2}{*}{$\begin{array}{l}\text { Matrix } \\
\text { Patterns }\end{array}$} & \multicolumn{3}{|c|}{ Premask Matrix } & \multicolumn{2}{|c|}{ Target Matrix } \\
\hline & $\begin{array}{c}\text { Matrix } \\
\text { Size }\end{array}$ & $\begin{array}{l}\text { Element } \\
\text { Size }\end{array}$ & $\begin{array}{c}\text { Element } \\
\text { Separation }\end{array}$ & $\begin{array}{l}\text { Matrix } \\
\text { Size }\end{array}$ & $\begin{array}{c}\text { Element } \\
\text { Size }\end{array}$ \\
\hline Triangle & $5^{\circ} 32^{\prime}$ & $56^{\prime}$ & $1^{\circ} 24^{\prime}$ & $4^{\circ} 36^{\prime}-5^{\circ} 32^{\prime}$ & $28^{\prime}$ \\
\hline Square & $4^{\circ} 48^{\prime}$ & $48^{\prime}$ & $1^{\circ} 12^{\prime}$ & $4^{\circ}-4^{\circ} 48^{\prime}$ & $24^{\prime}$ \\
\hline Hexagon & $7^{\circ} 12^{\prime}$ & $32^{\prime}$ & $48^{\prime}$ & $6^{\circ} 40^{\prime}-7^{\circ} 12^{\prime}$ & $16^{\prime}$ \\
\hline
\end{tabular}

two locations were never occupied by either the prime or the target (center up and center down). This rendered the number of possible prime/target locations (in this instance, four) equivalent for each target form geometry and, therefore, each of the three different display matrices. Details concerning the configuration of the three matrices are given in Table 1.

All the premask and target elements exhibited $40 \%$ specification; that is to say, of the continua between the premask crosses or stars, $40 \%(2 \times 20 \%)$ were physically specified by the presence of an inducing luminous contour, whereas the remaining $60 \%$ of the continua remained unspecified. In order to ensure that all the frames were equiluminant, each frame contained 500 illuminated pixels. This measure also served to maintain plot frequency as constant, and, in order to account for the fact that different stimulus image frames could comprise different numbers of illuminated pixels, pixels additional to those required for image presentation (to an overall number not exceeding 500) were illuminated in the lower left corner of the display and were rendered invisible by a small opaque patch glued to the display screen.

The individual premask frames and the target matrix frame were presented semistatically at a fixed $500-\mathrm{Hz}$ refresh frequency, to keep the image point luminance constant. The participants viewed the stimuli arranged around the center of the monitor screen at a distance of $57 \mathrm{~cm}$ (maintained via a chinrest). The experiment was conducted under controlled lighting conditions (mean screen surround luminance, $7.8 \mathrm{~cd} / \mathrm{m}^{2}$ ), with stimulus luminance maintained at $30 \mathrm{~cd} / \mathrm{m}^{2}$ upon a background field of $7.5 \mathrm{~cd} / \mathrm{m}^{2}$. The level of stimulus-background contrast (4:1) was kept consistent with that in previous studies, with stimulus luminance calibrated to avoid luminous distortion.

Design and Procedure. A trial started with a brief (300-msec) $250-\mathrm{Hz}$ computer-generated tone. Following a subsequent random delay of between 200 and $300 \mathrm{msec}$, the participants were presented with the premask matrix, which, after $600 \mathrm{msec}$, reduced to simple junctions by removal of redundant line segments (procedure illustrated in Figure 1). The participants were informed that the flickering matrix did not require a response but that they should fixate the center of the matrix and avoid eye movements during presentation. Upon removal of the redundant line segments, the participants were asked to produce an RT response, as rapidly and accurately as possible, to the presence or absence of a target Kanizsa-type triangle, square, or hexagon within the presented matrix of corner junctions. In the event of an erroneous response, feedback was provided through a second $(150-\mathrm{msec}) 100-\mathrm{Hz}$ computer-generated tone followed by a 500 -msec delay.

Experiment 2 used a within-subjects design, with the factors of target (present or absent), prime (interphase or intraphase), and geometrical form (triangular, square, or hexagonal). The target and prime factors were varied randomly within twenty 48-trial blocks. In order to avoid dramatic display size change from trial to trial, the factor of geometrical form was varied between blocks but was counterbalanced within conditions. The participants performed a total of 960 experimental trials.

\section{Results}

Those trials with error responses ( $4.1 \%$ of all the trials) were removed from the data prior to subsequent analyses. Error RTs tended to be longer than correct RTs overall, and analysis of the probability correct by RT revealed no significant correlation, which argues against the correct data being contaminated by accuracy-speed trade-offs. Examination of the correct RTs revealed a nonnormal distribution with pronounced positive skew. A Kolomogorov $D$ test showed the RT distribution to be approximately lognormal, and on this basis, subsequent analyses were conducted on the exponents of the means of log-transformed RT distributions (for supporting ideas, see Box \& Cox, 1964, 1982). Figure 5A presents the correct mean RTs (and their standard errors) as a function of target geometry, separately for each target (present or absent) $\times$ prime (intraphase or interphase) condition.

The correct mean RTs were examined by means of a repeated measures ANOVA with main terms of prime (intraphase or interphase premask element distribution), target (present or absent), and form geometry (triangle, square, or hexagon). Violations of the homogeneity of variance assumption were corrected by applying Huynh-Feldt epsilon adjustments (Huynh \& Feldt, 1976). All the main effects were significant [target, $F(1,13)=16.4, p<.01$; prime, $F(1,13)=54.8, p<.01 ;$ form geometry, $F(1.5,20.04)=$ $16.7, p<.01]$. Consistent with the possibility that there was some additional matrix search on target-absent trials, RTs were shorter to target-present than to target-absent matrices. RTs were shorter following intraphase premask presentation than following interphase premask presentation, whereas a Tukey's HSD test showed RTs to be shorter for hexagon and square matrices, as compared with triangle matrices (489, 492, and $511 \mathrm{msec}$, respectively). The elevated RTs to triangle targets may reflect the greater number of display locations (six, as opposed to four each in the square and hexagon matrices), even though in two of these locations a target was never presented. In agreement with the previous pattern of effects reported with this paradigm (i.e., Becker, Elliott, \& Lachmann, 2005; Elliott, Becker, Boucart, \& Müller, 2000; Elliott et al., 2006; Elliott, Herrmann, Mecklinger, \& Müller, 2000; Elliott \& Müller, 1998, 2000, 2001), the target $\times$ prime interaction was found to be significant $[F(1,13)=23, p<$ $.01]$, describing significant priming effects (i.e., the mean difference interphase minus intraphase premask RTs) that were confined to target trials (the mean priming effect in target-present conditions [and standard error of the mean] was 29 [4] msec, as compared with 6 [4] msec on targetabsent trials). On this basis and with a view to examining the priming effects specifically, further analyses were conducted on the target-present data only.

Analysis of the target-present data showed all the main effects to be significant, and of particular theoretical interest, the interaction between form geometry and prime was significant $[F(2,26)=9.49, p<.01]$. Further analyses were conducted to examine the relation between the magnitude of the priming effects and the subjective form complexity measured in Experiment 1 . The outcome of a linear regression analysis is shown in Figure 5B. Priming effects were found to increase as a monotonic function of the average measure of subjective form complexity derived from Experiment 1, with an approximately linear relation $\left(r^{2}=0.99, p<.05\right)$. 

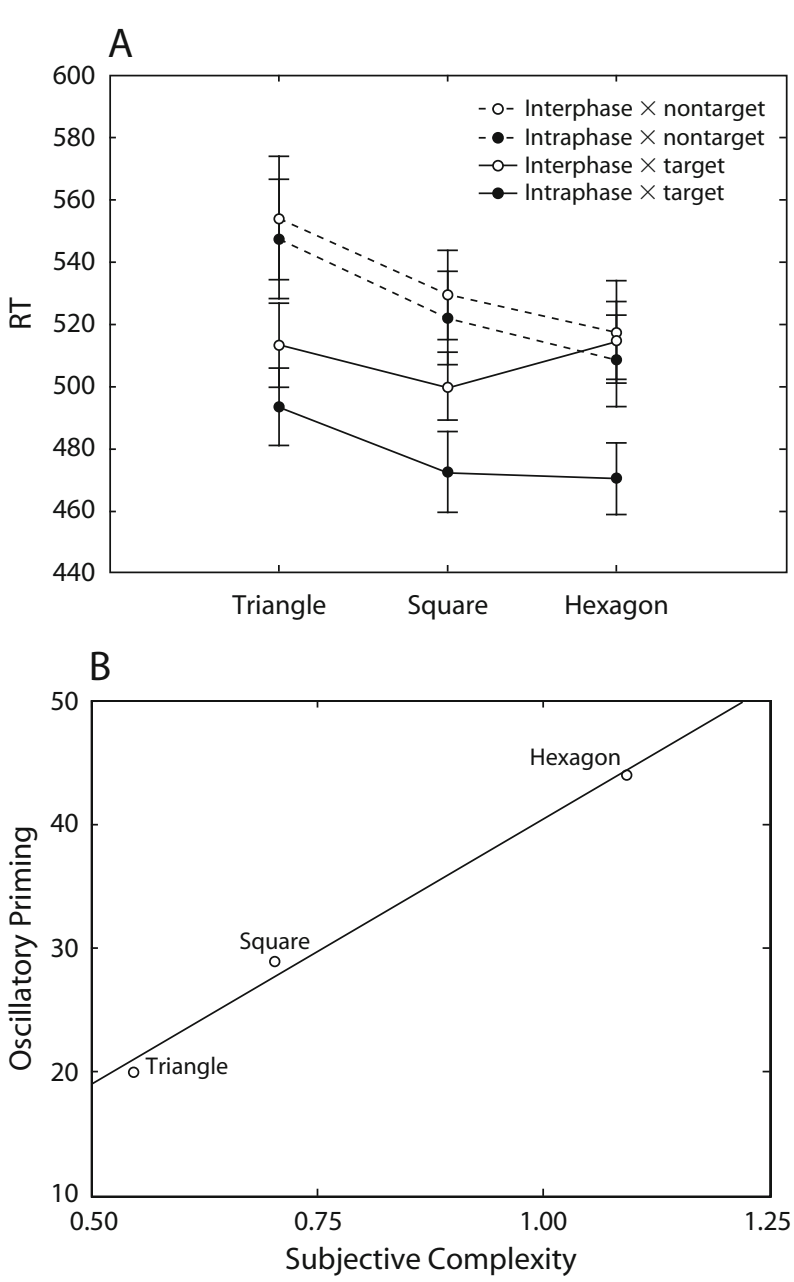

Figure 5. (A) Mean correct target-present and target-absent reaction times (RTs, in milliseconds; $\pm S E$ s) for intraphase and interphase conditions as a function of target form in Experiment 2. (B) Mean priming effects as a linear function of subjective form complexity.

Error analysis. An ANOVA of arcsine-transformed error data (with the same main terms as those for the RT data ANOVA) revealed that the trials on triangle forms produced slightly more errors than did the trials on square and hexagon forms $[F(2,26)=7.25, p<.01]$. Trials on which an intraphase premask was presented were found to produce slightly fewer errors than did trials on interphase premask trials $[F(1,13)=12.64, p<.01]$. However, the difference between miss rates and false alarms was nonsignificant $[F(1,13)=2.22]$. This indicates that the RT data were from the neutral response set and reduces the likelihood of contamination by speed-accuracy trade-offs. A number of two-way interactions were significant [form $X$ prime, $F(2,26)=4.43, p<.05$; form $\times$ target, $F(2,26)=$ $13.50, p<.01]$. Furthermore, post hoc tests $(p<.05)$ showed these were due mainly to lower false alarms in the hexagon conditions. The two-way prime $\times$ target interaction $[F(1,13)=16.12, p<.01]$ was due to more frequent miss rates following interphase premask conditions.

\section{Discussion}

Experiment 2 revealed that priming effects increased in magnitude in line with increasing subjective form complexity. On this basis, it might be assumed that the dynamic mechanisms responsible for oscillatory prime formation are, indeed, sensitive to prime/target structure. One should note, however, that form complexity was not directly related to mean RT performance, which may, instead, relate more specifically to design asymmetries in matrix construction (accounting for some of the difficulty in detecting triangles, relative to the other target forms) and the relative size of the target forms (hexagons were detected both more quickly and more easily, as is evident from both the RT and the error data). In Experiment 2, subjective form complexity seems to have had an influence only on the magnitude of priming.

However, the results of Experiment 1 also showed that subjective complexity increased as a monotonic function of the number of sides for triangles, squares, and hexagons aside from pentagons (Figure 3A). Thus, the linear relationship between priming and form complexity may reduce to the relationship between priming and the number of sides. It seems impossible to dissociate the two explanations on the subset of the triangle, square, and hexagon, since number of sides and subjective complexity are almost equivalent measures. However, these two alternative explanations may be dissociated from each other by including the pentagon form, which was shown in Experiment 1 to be considered of relatively high complexity.

Another limitation of Experiment 2 was that although prime/target forms were varied, as in Elliott and colleague's previous experiments in which a square matrix held square primes/targets, the prime target forms were predictable on the strength of matrix design. In Experiment 2, matrix design was confined to forms that permitted perfect tiling, and although this was consistent with the matrix design used previously, it precluded the use of pentagons. Although the relative dimensions of the three forms were controlled for in the matrices employed in Experiment 2,3 the data suggest various configurational effects on both RTs and errors, and the influence of these effects on priming cannot be entirely ruled out. In addition, it is possible that priming may relate to the predictability of the target form, especially given the presence during priming-stimulus presentation of coactive sources under anterior and posterior electrodes (Conci et al., 2004; Elliott et al., 2003). This is equivalent to suggesting that some significant proportion of priming emerges as a function of identity priming (i.e., the preactivation of square, triangle, or hexagon templates in visual shortterm memory), which might come about not through the dynamics of prime formation, but as a result of simply second-guessing the prime form on the basis of the configuration of the premask matrices as a whole.

Thus, given a need to dissociate the effects of form complexity from the number of sides, which introduces the necessity to include pentagons to provide a deciding measure of priming, and the need to remove potential constraints inherent in matrix design, which seem to preclude 
an unambiguous interpretation of the priming effects, Experiment 3 was designed to cross-validate with the results of Experiment 2 by introducing a uniform mixture matrix, in which matrices were of identical dimensions and within which any one of four possible primes/targets might be presented, including prime/target pentagons.

\section{EXPERIMENT 3}

Whereas Experiment 2 dealt with the interaction between form complexity and oscillatory priming, using tiled matrices, Experiment 3 was an attempt to examine those effects by using a uniform mixture matrix. The matrix was designed to accommodate four different possible target forms alongside additional distractor elements. Unlike in the previous experiment, in which forms were tightly embedded within perfectly tiled matrices, the forms used in the mixture matrix were distributed loosely across one of four independent locations. Four form conditions were examined in Experiment 3, corresponding to triangles, diamonds (squares), pentagons, and hexagons.

\section{Method}

Participants. Eleven participants, all with normal or correctedto-normal vision, participated in Experiment 3 (5 of them male; mean age, 26.7 years). The participants were naive as to the precise experimental conditions presented in the experiment and were paid at a rate of $€ 8 / \mathrm{h}$.

Stimuli and Procedure. As in Experiment 2, the stimulus display (illustrated in Figure 6) consisted of a matrix of premask elements distributed across four sequentially presented image frames. The sequence of premask image frames was presented for $600 \mathrm{msec}$ and at a frame frequency of $40 \mathrm{~Hz}$, after which the premask display transformed into a static display of corner junctions. Unlike in Experiment 2 , the premask display was a single mixture matrix comprising 24 elements distributed around the center of the oscilloscope screen, so that 3 elements were positioned at the apexes of a regular triangle, 4 at the apexes of a diamond, 5 at the apexes of a pentagon, and 6 at the apexes of a hexagon. These four possible forms were of identical circumference, plotted around identical circular coordinate systems, which subtended $2^{\circ} 40^{\prime}$ of visual angle at a viewing distance of $57 \mathrm{~cm}$. The four possible prime/target forms occupied each of the four display quadrants (which were separated by $2^{\circ} 40^{\prime}$ of visual angle) with equal probability, but with the precise configurations trial-wise randomized in advance of the experiment. The remaining 6 display elements were positioned randomly around the display, with a minimum element-element separation of $48^{\prime}$ to $1^{\circ} 24^{\prime}$.

Because the four possible geometrical forms have different angular specifications at their apexes, perfect element-element collinearity would require a star with 10 line segments. The perceptual effects of this would have been of a very bright dot with no discernible inducer information. A pilot experiment was conducted to test the effects of local inducer line orientation on priming, the results of which confirmed that small angular deviations from perfect collinearity are of very little significance for priming. ${ }^{4}$ Consequently, in order to equalize any random effects arising from particular inducer specifications, while at the same time maintaining premask and target displays as equiluminant, a star with three line segments was used as the premask inducer (Figure 6). The polar angles of the three line segments were set to $17^{\circ}, 77^{\circ}$, and $137^{\circ}$, respectively, in order to render them equipotent with respect to the different angular displacements of the inducers in the 3-, 4-, 5-, and 6-element target forms. The star elements subtended $32^{\prime}$ of visual angle, and separation from their nearest neighbors varied from $48^{\prime}$ to $1^{\circ} 24^{\prime}$.

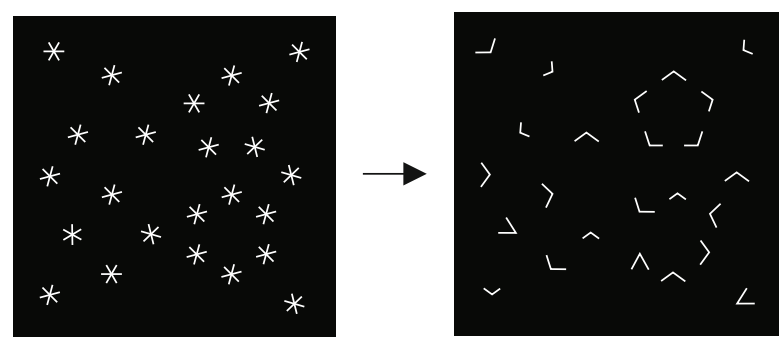

Figure 6. Schematic illustration of premask and target matrices in Experiment 3.

As in Experiment 2, the 24-element premask display comprised four sequential frames, with each frame containing between 3 and 7 unique premask elements. The whole premask matrix display subtended $8^{\circ} 16^{\prime} \times 8^{\circ} 16^{\prime}$. On $50 \%$ of the occasions, the target forms were preceded at their presentation locations by the intraphase presentation of a matching number of premask elements arranged in the corresponding geometrical form (the intraphase condition), as compared with an interphase condition in which the spatiotemporal organization of the premask elements was fully randomized. A signal detection experiment similar to that conducted in Experiment 2 (see note 2 ) showed that 12 participants ( 7 of them female; mean age, 27.4 years; all with normal or corrected-to-normal vision; each produced 320 trials for all conditions) were unable to discern the intraphasic or interphasic structure of a given premask frame ( $A_{z}$ varied from 0.473 to 0.559 between participants, $M=0.504$, $S E=0.008)$. Targets were presented on $50 \%$ of the trials, and on the other $50 \%$ of the trials, no grouping was presented in the final target display. The target matrix subtended $8^{\circ}-8^{\circ} 16^{\prime} \times 8^{\circ}-8^{\circ} 16^{\prime}$ of visual angle. The elements making up the target matrix comprised the junctions of four possible Kanizsa-type target forms - that is, three junctions subtending $28^{\prime}$ for triangles, four junctions subtending $24^{\prime}$ for squares, five junctions subtending $16^{\prime}$ for pentagons, and six junctions subtending $16^{\prime}$ for hexagons. Note that in order to balance target conspicuity across the four forms, each possible target was specified by a luminous contour to $40 \%$ of the continuances between apexes.

Experiment 3 used a within-subjects design, with the factors of target (present or absent), prime (interphase or intraphase), and geometrical form (triangle, square, pentagon, or hexagon). In the targetpresent trials, only one possible form out of the four was presented (all the forms being presented with equal probability in the experiment as a whole). The combinations of target positions and forms were employed with equal probability; that is, each possible form appeared equally at each of the four possible locations. In the intraphase premask condition, the priming frame was the first frame of the four-frame sequence. Each participant was presented with 1,024 trials within sixteen 64-trial blocks. The experimental procedure was the same as that described for Experiment 2.

\section{Results}

As in Experiment 2, trials with an erroneous response were removed from the data prior to subsequent analyses ( $7 \%$ of all the trials). Error RTs tended to be longer, overall, than correct RTs, and analysis of the probability correct by RT revealed no significant correlation, arguing against the correct data's being contaminated by accuracy-speed trade-offs. An examination of the raw correct RT data revealed nonnormal distributions with pronounced positive skew. A Kolomogorov $D$ test showed the RT distributions to be approximately lognormal, and on this basis, subsequent analyses were conducted on the exponents of the 
means of log-transformed RT distributions. Figure 7A presents the correct mean RTs (and their standard errors) as a function of target geometry and separately for each target (present or absent) $\times$ prime (intraphase or interphase) condition.

A repeated measures ANOVA was conducted over all factors: target, prime, and geometrical form. All the main effects were significant [target, $F(1,10)=16.56, p<.01$; prime, $F(1,10)=20.80, p<.01$; form, $F(3,10)=19.79$, $p<.01]$. As in Experiment 2, and consistent with the possibility that there was some matrix search on target-absent trials, RTs were shorter to target-present than to targetabsent matrices. In addition, RTs were shorter following intraphase premask than following interphase premask presentation. Finally, RTs tended to be shorter for forms with more sides, although this conclusion can be reached only with respect to the target-present trials (see Figure $7 \mathrm{~A}$ ) and was true because target detection tended to be easier when the proportion of grouping parts increased. The interaction between prime and target was significant
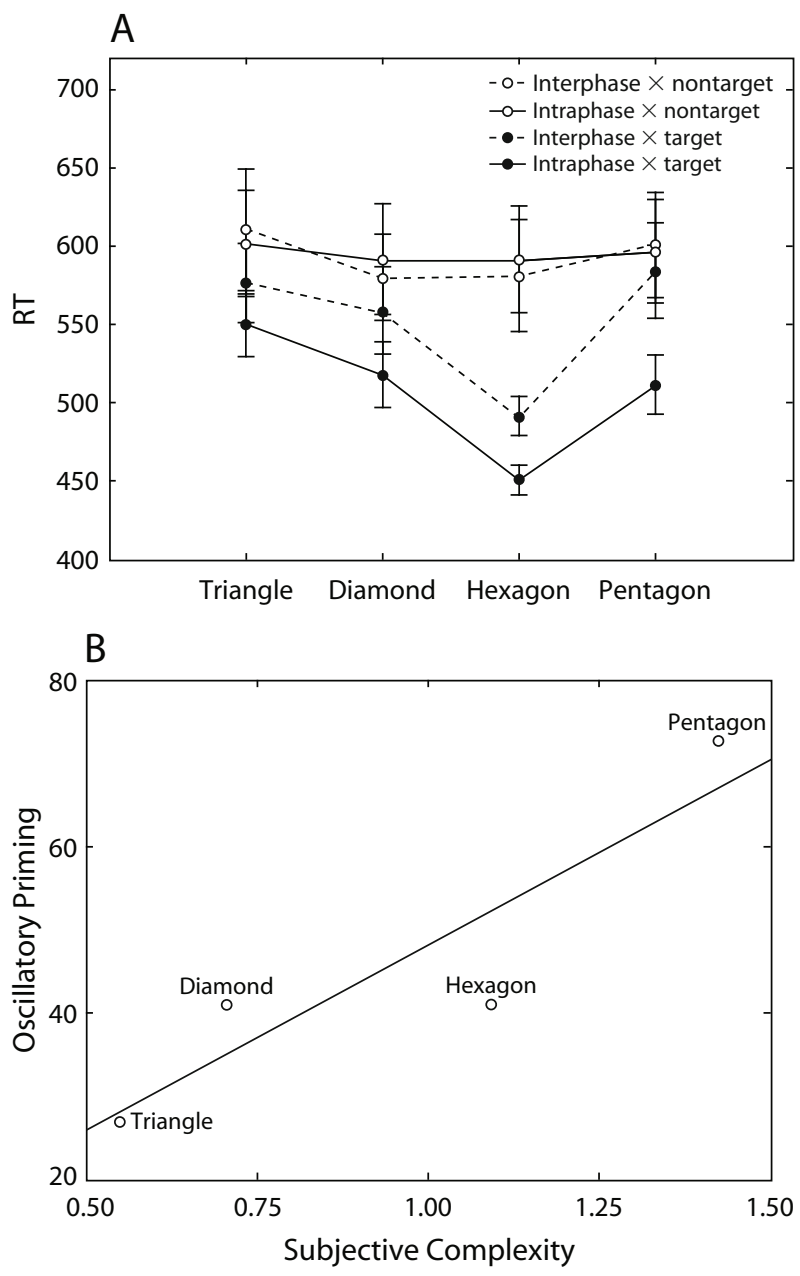

Figure 7. (A) Mean correct target-present and target-absent reaction times (RTs, in milliseconds; $\pm S E$ s) for intraphase and interphase conditions as a function of target form in Experiment 3 . (B) Mean priming effects as a linear function of subjective form complexity.
$[F(1,10)=15.98, p<.01]$, with significant priming effects confined to the target trials (mean RT enhancements for intraphase, relative to interphase, premask conditions [and standard errors of the means] were 45 [7] msec for the target-present trials, as compared with -4 [5] msec for the target-absent trials).

On the basis of the significant target $\times$ prime interaction and in order to examine the relationship between prime and form, a further ANOVA was conducted on the target-present data only. All the main effects were significant [prime, $F(1,10)=20.04, p<.01$; geometrical form, $F(3,30)=17.20, p<.01]$, and of principle theoretical interest, the interaction between geometrical form and prime was significant $[F(3,30)=3.58, p<.05]$. This is suggestive of an increase in the magnitude of priming commensurate upon an increase in the number of sides possessed by the target. Critically, the ambiguous relation between priming and the number of sides seems to be resolved in Experiment 3: The number of sides of the prime/target form was found not to explain variations in the magnitude of priming $\left(r^{2}=.24, p>.1\right)$, which were found, instead, to increase with an approximately linear relation as a monotonic function of the average measure of subjective form complexity derived from Experiment 1 (see Figure 7B; $r^{2}=.824, p=.09$ ).

Error analysis. An ANOVA of arcsine-transformed error data revealed that the miss rates were higher than the false alarm rates $[F(1,10)=36.5, p<.01]$. Although this effect, alongside the general increase in errors, relative to Experiment 2, suggests that target detection is more difficult in the mixture matrix than in the regularly tiled matrices, it nonetheless argues against the presence of fast guess responses and, by extension, against the RT data's being contaminated by speed-accuracy trade-offs. Furthermore, trials on which the targets were triangles showed an overall higher error rate than did trials with the other forms, whereas trials upon which hexagons were the targets showed the lowest error rates $[F(3,30)=27.11, p<.01]$. This suggests, as with regularly tiled matrices, that the longer RTs to triangles may have been due to their relative inconspicuity [supported by the two-way form $\times$ target interaction- $F(3,30)=10.8, p<.01-$ which achieved significance due to some difficulty in the detecting of triangle], whereas the shorter RTs to hexagons may have been due to their relative size. The two-way prime $\times$ target interaction was also found to be significant $[F(1,10)=$ $21.39, p<.01]$, and post hoc Tukey HSD tests $(p<.05)$ revealed that targets followed by interphase premask matrices produced more errors than did other conditions.

\section{Discussion}

The results of Experiment 3 showed that priming effects and the interaction between priming and geometrical form were obtained when the primes were not uniquely predictable and when the prime/target forms were embedded in a mixture matrix. This outcome corroborates the findings of Experiment 2, in that priming increased with an increase in the subjective complexity of the target, and thus tends to argue against a strict identity-priming account for oscillatory priming. 
The most important result in Experiment 3 was that, akin to the measures of subjective complexity for regular forms (Experiment 1) in which pentagons were considered to be the most complex of the four forms examined, in Experiment 3 the maximum priming effect was found for pentagon targets. This effect serves to rule out one possibility raised from the outcome of Experiment 2, that the magnitude of priming is a function of the number of sides of the form, and raises the intriguing outcome that priming is increased for forms that are more difficult to detect and that are classified as perceptually more complex. These results are also suggestive of the interpretation that a form of wholistic complexity is activated during premask presentation. This is consistent with the minimum principle, according to which the whole system gives more engagement to more complex processing scenarios, and this may explain why oscillatory priming increases as prime/target form complexity increases.

The results of both Experiments 2 and 3 suggest that the minimum principle influences the magnitude of oscillatory priming. As was shown in Experiment 1, form regularity is also a feature of grouping based on the minimum principle, and it is still unclear whether the relationship between priming and complexity can be extended to irregular forms - in other words, whether the relation between complexity and the magnitude of priming is in some way related to the regularity of the forms concerned. Experiment 4 was designed to explore these relationships.

\section{EXPERIMENT 4}

Experiment 4 was conducted with the aim of evaluating the outcome of Experiments 2 and 3 with irregular prime/ target forms. Four different target forms - irregular triangle, quadrilateral, and five- and six-sided forms - were used in Experiment 4. The shapes of the four irregular forms were set the same as those in Experiment 1, in order to use the subjective complexity values obtained from Experiment 1 .

\section{Method}

The design and procedure in Experiment 4 were identical to those in Experiment 3, with the following exceptions.

Participants. Fourteen participants, all with normal or correctedto-normal vision, participated in Experiment 4 (4 of them male; mean age, 26.7 years). The participants were paid at a rate of $€ 8 / \mathrm{h}$.

Stimuli and Procedure. A 24-element mixture matrix was employed, although the positions of the elements differed from those in Experiment 3 (see Figure 8). The positions of 6 distractor elements and the circular coordinate systems of four possible forms remained in the same positions as in Experiment 3. The remaining 18 elements were also positioned on the circular coordinate systems (which subtended $2^{\circ} 40^{\prime}$ of visual angle at a viewing distance of $57 \mathrm{~cm}$ ), but the horizontal polar angles were changed to the settings in Experiment 1 (see Figure 2A). A four-line star with a visual angle of $32^{\prime}$ was used as prime inducer. In order to better render them equipotent with respect to the different angular displacements required for specification of the three-, four-, five-, and six-element prime/target forms, the polar angles of four line segments of the star were $30^{\circ}, 45^{\circ}, 120^{\circ}$, and $150^{\circ}$, respectively.

Target displays subtended $8^{\circ} 16^{\prime} \times 8^{\circ} 16^{\prime}$ and consisted of the junctions of four possible target forms - that is, three junctions that go together to form an irregular triangle and the four, five, and six junctions that go together to form an irregular quadrilateral and

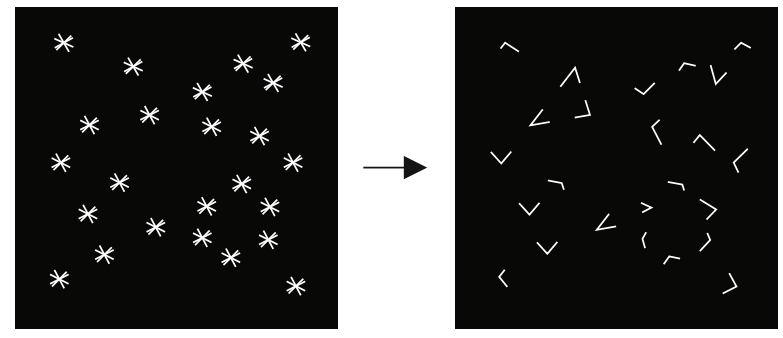

Figure 8. Schematic illustration of premask and target matrices in Experiment 4.

five-sided and six-sided forms, respectively. The length of the line segments inside each junction was calculated individually, so that the potential prime and target were always specified for $40 \%$ of the apex-apex continua. A pilot signal detection task experiment similar to that conducted for Experiment 2 (see note 2) showed that 12 participants ( 7 of them female; mean age, 27.4 years; all with normal or corrected-to-normal vision; each produced 320 trials for all the conditions) were unable to discern the intraphasic or interphasic structure of a given premask frame $\left(A_{z}\right.$ varied from 0.458 to 0.547 among participants; $M=0.501, S E=0.008$ ).

\section{Results}

Trials on which an error was made were removed from the data prior to subsequent analyses ( $7.7 \%$ of all the trials). Error RTs tended to be longer, overall, than correct RTs, and an analysis of the probability correct by RT revealed no significant correlation, which argues against the correct data's being contaminated by accuracy-speed tradeoffs. An examination of the raw correct RT data revealed nonnormal distributions with pronounced positive skew. A Kolomogorov $D$ test showed the RT distributions to be approximately lognormal, and on this basis, subsequent analyses were conducted on the exponents of the means of logtransformed RT distributions. Figure 9 presents the correct mean RTs (and their standard errors) as a function of target form, separately for each target (present or absent) $\times$ prime (intraphase or interphase premask) condition.

The mean correct RT data were examined by means of a repeated measures ANOVA with main terms of target (present or absent), prime (intraphase or interphase), and geometrical form (irregular triangle, quadrilateral, pentagon, or hexagon). All the main effects were significant [target, $F(1,13)=17.21, p<.01$; prime, $F(1,13)=73.28, p<$ .01 ; geometrical form, $F(3,39)=67.72, p<.01]$. As was expected, RTs were shorter on target-present than on targetabsent trials and were shorter following intraphase premask than following interphase premask presentation. RTs increased as the number of sides overall decreased. Consistent with the results of previous studies, the significant prime $X$ target interaction was due to priming effects being confined to target-present trials $[F(1,13)=39.18, p<.01]$. The mean priming effect (and the standard error of the mean) was 50 (5) msec for target-present trials, as compared with 1 (4) msec for target-absent trials. The three-way interaction was nonsignificant.

As in the previous experiments, the relationship between prime and form was examined using a further ANOVA conducted on the target-present data only. The two main effects 


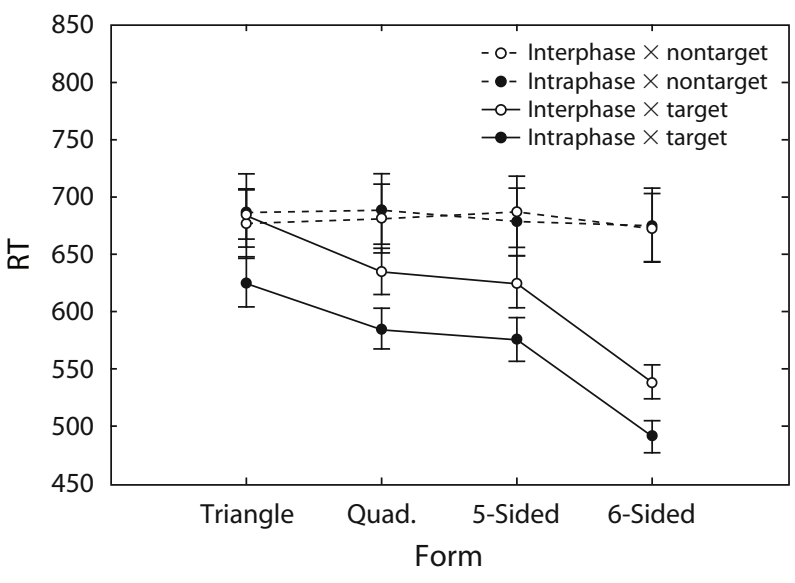

Figure 9. Mean correct target-present and target-absent reaction times (RTs, in milliseconds; $\pm S E$ s) for intraphase and interphase conditions as a function of target form in Experiment 4 .

were significant [prime, $F(1,13)=62.82, p<.01$; geometrical form, $F(3,39)=56.25, p<.01]$. However, critically, unlike in either Experiment 2 or 3, the interaction between prime and form was nonsignificant $[F(3,39)=0.49$, n.s.]. This indicates that priming was statistically equivalent in magnitude, irrespective of variations in the complexity or number of sides of the prime/target form.

Error analysis. The mean error rate was $7.7 \%$ across all the conditions. An ANOVA of arcsine-transformed error data revealed that trials in the interphase premask condition showed slightly but significantly higher errors than did those in the intraphase condition $[F(1,13)=6.92, p<$ .05]. Furthermore, and consistent with both Experiments 2 and 3 , trials on which the targets were triangles were found to show more errors than did trials on which the targets were quadrilaterals. Trials on which targets were five-sided and six-sided targets showed the lowest error rates $[F(3,39)=42.55, p<.01]$. [Note that, as in Experiment 3 , the form $\times$ target interaction was significant$F(3,39)=48.4, p<.01$ - and was due to a higher miss rate for triangle targets.] The two-way prime $\times$ target interaction was significant $[F(1,13)=11.72, p<.01]$, and as was revealed by post hoc Tukey tests $(p<.05)$, this was due to more errors following interphase premask presentation when combined with target presentation.

\section{Discussion}

The results of Experiment 4 confirmed that priming effects could be recorded even when prime/target forms were irregular; however, the absence of a significant interaction between prime and geometrical form indicates a major difference between the results of Experiment 4 and those of Experiments 2 and 3. In the previous experiments, the magnitude of priming increased almost linearly with increases in a measure of subjective complexity. Although this relation holds for regular forms, Experiment 4 showed that for irregular forms, priming effects remain constant irrespective of subjective complexity. Invariant priming effects may indicate that a very general and nonspecific preactivation follows intraphase premask element presentation. However, it also suggests that this preactivation is not sensitive to irregular form per se.

Thus, this experiment shows that irregularity breaks down the relation between prime and complexity. This breakdown has two possible explanations. One concerns the operation of the minimum principle. According to the minimum principle, the whole system seeks to reduce its engagement to a minimum, and thus, during the short premask presentation time, the visual system reduces its engagement to complex forms beyond some (unknown) level of complexity and waits for the appearance of the target and task execution. Another possibility concerns a potential imbalance in the search task for the different figures. In spite of irregularity, almost any three adjacent elements can construct an irregular triangle. Consequently, the number of possible irregular triangles is greater than the number of possible six-sided forms, leading to an unequal number of potential target feature locations in the premask/target matrix between the different irregular forms. This possibility was inherited from the design of the matrix in Experiment 4, and as a consequence, in Experiment 5, the matrix involved a rearrangement and equalizing of the number and position of elements referring to the different potential prime/target forms.

\section{EXPERIMENT 5}

Experiment 5 employed irregular prime/target forms and altered the positions of elements in the mixture matrix to form four distinct subregions at which a prime/target might be presented, although these subregions exhibited no specific arrangement.

\section{Method}

The design and procedure in Experiment 5 were identical to those in Experiment 4, with the following exceptions.

Participants. Fourteen participants, all with normal or correctedto-normal vision, participated in Experiment 5 ( 3 of them male; mean age, 25.4 years). The participants were paid at a rate of $€ 8 / \mathrm{h}$.

Stimuli and Procedure. A 24-element mixture matrix was employed, although the arrangement of the elements differed from that used in Experiment 4 (see Figure 10). In this instance, 24 elements were divided into four subgroups of 6 elements, which were positioned around a circle. Each of these subgroups subtended $2^{\circ} 40^{\prime}$ of visual angle at a viewing distance of $57 \mathrm{~cm}$, and the polar angles of the 6 elements were fixed at $0^{\circ}, 30^{\circ}, 120^{\circ}, 160^{\circ}, 210^{\circ}$, and $270^{\circ}$, respectively. With such positioning, the four irregular forms introduced in Experiment 4 could be employed in Experiment 5, although it was a priori impossible to tell at which location which form would be presented. Target displays subtended $8^{\circ} \times 8^{\circ}$ and consisted of the junctions of four possible target forms. The specifications of four irregular target forms were identical to those in Experiment 4. The conditions and procedures were the same as those in Experiment 4. A pilot signal detection experiment similar to that conducted for Experiment 2 (see note 2) showed that 12 participants ( 7 of them female; mean age, 27.4 years; all with normal or corrected-to-normal vision; each produced 320 trials for all conditions) were unable to discern the intraphasic or interphasic structure of the priming stimuli with the mixture matrix used in Experiment $5\left(A_{z}\right.$ varied from 0.45 to 0.552 between participants; $M=0.508, S E=0.008$ ). 


\section{Results}

RTs on trials on which a response error was made $(6.1 \%$ of all the trials) were removed from the data prior to subsequent analyses. Examination of the raw correct RT data revealed nonnormal distributions with pronounced positive skew. A Kolomogorov $D$ test showed the RT distributions to be approximately lognormal, and on this basis, subsequent analyses were conducted on the exponents of the means of log-transformed RT distributions. Figure 11 presents the correct mean RTs (and their standard errors) as a function of target form, separately for each target (present or absent) $\times$ prime (intraphase or interphase premask) condition.

The mean correct RT data were examined by means of a repeated measures ANOVA with main terms of target (present or absent), prime (intraphase or interphase), and geometrical form (irregular triangle, quadrilateral, pentagon, or hexagon). All the main effects were significant [target, $F(1,13)=37.92, p<.01$; prime, $F(1,13)=$ $34.14, p<.01$; geometrical form, $F(3,39)=71.91, p<$ $.01]$. As was expected, RTs were shorter on target-present than on target-absent trials and were shorter following intraphase premask than following interphase premask presentation. Consistent with previous studies, the significant prime $\times$ target interaction was due to priming effects' being confined to target-present trials $[F(1,13)=$ $17.76, p<.01]$. The mean priming effects (and standard error of the mean) was 33 (5) msec for target-present trials, as compared with 1 (7) msec for target-absent trials. The three-way interaction was nonsignificant. As in the previous experiments, the relationship between prime and form was examined using a further ANOVA conducted on the target-present data only. The two main effects were significant [prime, $F(1,13)=47.67, p<.01$; geometrical form, $F(3,39)=100.69, p<.01]$. However, as in Experiment 4 , the interaction between prime and form was nonsignificant $[F(3,39)=0.93$, n.s. $]$.

Error analysis. An ANOVA of arcsine-transformed error data revealed that error rates increased with a decrease in the number of sides of the target forms $[F(3,39)=$ $36.26, p<.01]$. Furthermore, interphase premask trials showed slightly more errors, overall, than did intraphase trials $[F(1,13)=26.85, p<.01]$. Miss rates were higher than false alarm rates $[F(1,13)=69.13, p<.01]$, and the two-way form $\times$ target interaction, which was significant $[F(3,39)=40.61, p<.01]$, was attributable mainly to dif-

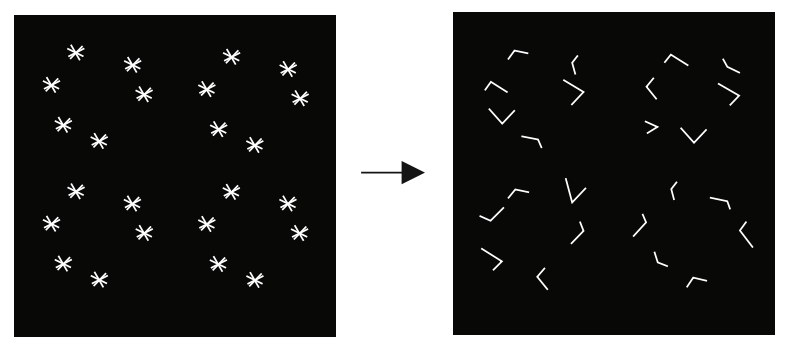

Figure 10. Schematic illustration of the premask and target matrices in Experiment 5.

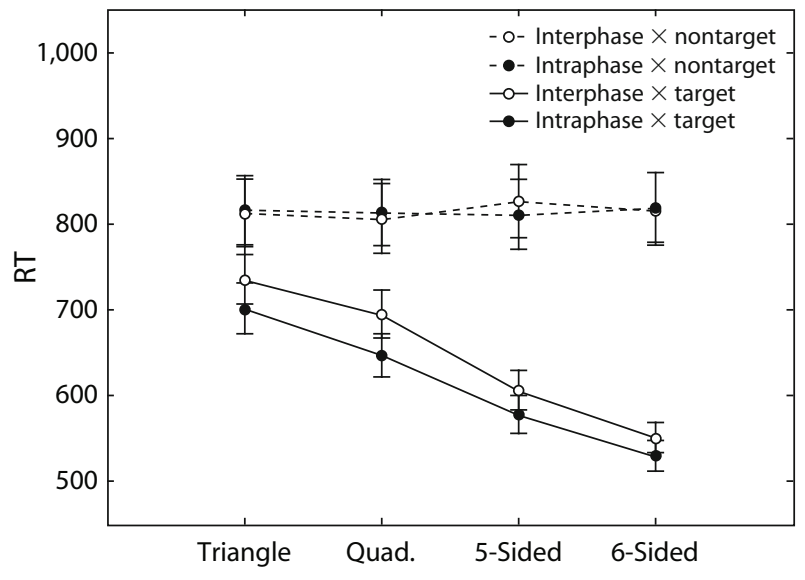

Figure 11. Mean correct target-present and target-absent reaction times (RTs, in milliseconds; $\pm S E s)$ for intraphase and interphase conditions as a function of target form in Experiment 5.

ficulties in detecting triangular targets (Tukey HSD, $p<$ $.05)$. As in Experiments 3 and 4, the two-way prime $\times$ target interaction was significant $[F(1,13)=9.59, p<.01]$, and as was revealed by post hoc Tukey tests $(p<.05)$, this was due to more errors following interphase premask presentation when combined with target presentation.

\section{Discussion}

Priming effects that do not vary with variations in subjective complexity are similar to those found in Experiment 4 , indicating that for conditions under which prime/target forms are both irregular and completely unpredictable, the relation between subjective complexity and priming does not obtain.

\section{GENERAL DISCUSSION}

The aim of this research was to explore whether or not priming performance varied with variations in figural complexity. By extension, a second aim was to determine whether evidence exists to support the idea of neuronal synchronization as one measure of the minimum principle. In contrast to the previous studies of Elliott and Müller $(1998,2000,2001)$, in which the priming stimuli were squares, the experiments reported here used a number of regular and irregular polygonal forms. In Experiment 1, a measure of the Prägnant quality of different polygons was obtained, with the aim of deriving a model of subjective complexity against which the data in four subsequent priming experiments could be compared. Experiments 2 and 3 manipulated the display matrices (using tiling and uniform mixture matrices) with various target forms (regular triangles, squares, pentagons, and hexagons). As was expected, target detection was expedited by intraphase premask presentation, which was consistent with the results of all the previously reported studies in which this paradigm was used. The most important outcomes of Experiments 2 and 3 were the relations between the magnitude of priming and subjective complexity: Priming effects increased as a linear function of the subjective 
complexity of regular prime/target forms. This indicates that a pattern of prime activity corresponding to a regular Gestalt becomes active during premask matrix presentation. Taking into consideration that the participants were not aware of and did not "see" the priming stimulus, a necessary entailment of this observation is that a pattern of activity develops, in the absence of direct perception, that nonetheless relates directly to the perceived structural complexity of the visual form concerned. In Experiments 4 and 5, four irregular forms (corresponding to irregular three-, four-, five-, and six-sided polygons) were tested in a mixture matrix, and in contrast to the results of Experiments 2 and 3 , priming effects remained constant, irrespective of form complexity. Taken together, the results of Experiments 2-5 indicate that oscillatory priming is sensitive to Prägnant quality when the prime/target forms are regular or predictable. Conversely, when the forms are irregular or unpredictable, they may come to be processed by different means, with redundant variations in complexity being ignored for the purposes of processing and prime formation.

It was suggested in Experiment 1 that form complexity was related not only to the number of sides, but also to symmetry, a suggestion appealing to the studies conducted by Garner, Palmer, Leeuwenberg, and others (see Arnoult, 1960; Garner, 1970, 1974; Leeuwenberg, 1968; Mavrides \& Brown, 1969; Palmer, 1991). By extension to these studies, the pairwise comparison in Experiment 1 shows that subjective complexity exhibits a power law relation with respect to the number of independent sides and levels of symmetry. Symmetry level acts as a power order of subjective complexity, which indicates that subjective complexity may increase dramatically when forms become less symmetrical. This is in accord with Leeuwenberg's minimum coding principle that less symmetrical forms require more information (bits) for encoding the whole form.

Perhaps not unexpectedly, in Experiment 1, the irregular forms were significantly harder to remember and reproduce than were the regular forms. A comparison of the differences in subjective complexity between the irregular and the regular forms shows that the differences between forms with the same number of sides increase as a linear function of the number of sides (adjusted $r^{2}=.92, p<$ .05 ). This suggests that subjective complexity may comprise two components. The first reflects the form category structure, which uses regular form as the category prototypes. The second component is an additive factor of complexity and may reflect the dissimilarity distance from the regular form. Feldman (2000) showed that mental models of shape bias toward regular forms, which supports the idea that the regular form is a fundamental component of complexity. The regular shape is also considered by some researchers to be a fundamental feature that occurs very early in visual processing (Donnelly, Humphreys, \& Riddoch, 1991; Pomerantz \& Pristach, 1989). This suggests that one component of priming may involve mechanisms ordinarily responsible for the low-level segmentation of the visual scene. On the other hand, dissimilarity from the prototype requires more specific features, which are un- able to be successfully or efficiently parsed during early processing.

Irregularity was introduced in the priming tasks in Experiments 4 and 5 and, as has been mentioned, in contrast to the results with regular forms, priming did not vary with varying complexity and, instead, remained at a constant magnitude. Considering the already introduced idea that when visual forms are processed for structural complexity, more than one means are used, it seems reasonable to suppose that the heuristic element in the coding of irregular forms in Experiment 1 was minimally engaged, if it was engaged at all, to resolve the structural organization of the priming stimulus in Experiments 4 and 5. As has been mentioned above, the dissimilarity component requires specific features. This means that the absence of variation in priming in Experiments 4 and 5 may relate to the lack of engagement of specific irregular feature processing.

At this stage, it is perhaps relevant to note that priming was obtained in all the experiments and irrespective of regularity or other factors, such as global changes to matrix design. Looking at the mean priming effects in Experiment 5, it might be claimed that even if the participants undertook, on average, a two-location search for the target (i.e., assuming serial self-terminating target search), the average priming effect was maintained constant at around $37 \mathrm{msec}$. This compares well with a mean priming effect of $33 \mathrm{msec}$ in Experiment 2, which, like Experiment 5, involved four potential target locations (except for triangle matrices, for which RTs were relatively high and priming relatively weak). In both of these experiments, the factor of which type of target was presented was controlled for; it was either obvious (Experiment 2) or impossible to guess in advance (Experiment 5) and, thus, may have been redundant. This redundancy may account for the $\sim 10$-msec reduction in prime efficiency, relative to Experiments 3 and 4, in which the mean priming effects were of 45 and $50 \mathrm{msec}$, respectively, and potential target forms may have been second-guessed.

In general, priming seems to increase with increasing complexity. This is illustrated, for example, by the effects of redundancy in Experiments 2 and 5, which also illustrate priming without prime/target Prägnanz as a factor for consideration. In addition, Experiments 2 and 3 show that primes are generally of increased magnitude for forms that are more difficult to detect and are classified as perceptually more complex. Equated with varying levels of neuronal activation, prime activation might thus be considered to increase with increasing form complexity and to be reduced for simpler forms - a formulation that makes perfect sense in the context of the minimum principle, which considers high activation to be associated with relatively complex processing tasks and low activation to be associated with tasks of lesser complexity.

Returning to physiology, one interim conclusion arising from this discussion is that unless subject to modification or modulation, the type of visual-cortical synchrony responsible for coding the intraphasic premask may not necessarily come to be endowed with the requisite figural code to directly bring about variation in Prägnanz quality. In other words, taken in isolation from other factors, low-level synchrony may vary with form complexity but 
does not do so automatically and directly on the strength of stimulus-driven information alone. This raises the issue of identifying the basis on which intraphasic priming varies with subjective complexity, and ultimately, this issue refers to which mechanism we can attribute variations in the perceived complexity of visual forms. Following the line of argumentation developed in this article, we can conclude that when the form of the prime can be secondguessed with a certain degree of probability, the magnitude of priming varies with the Prägnant quality of the form. On this basis, it seems reasonable to suppose that candidate task-relevant forms are activated and maintained as a form of visual short-term memory during premask matrix presentation. Variations in priming as a function of varying Prägnant quality may thus be considered to come about as a function of operations in visual short-term memory on the basis of which the spatiotemporal pattern of activation across the prime becomes modified. Furthermore, and as was suggested in the previous discussion, this modification follows the minimum principle. In the light of the preceding discussion, we might conclude that the reduction of activation to a minimum when forms exhibit no Prägnant quality (Experiment 5) may not be achieved by virtue of precisely the same mechanism as is the reduction in activation associated with a form or range of forms for which Prägnanz may at last be guessed. In this sense, and given that we appeal to the minimum principle as an overarching level of description for the function of dynamic systems, we must acknowledge that in this case, it describes at least two different means of processing very similar stimulus materials. The resulting model of form-based intraphasic priming presupposes the interaction of memory and a stimulus-driven synchrony code, which is quite consistent with the idea that intraphasic priming arises as a function of the coactivation of early and late visual mechanisms by means of a recurrent network of activation (see Conci et al., 2004; Elliott et al., 2003; Elliott \& Müller, 1998, 2000). In this instance, the EEG data suggest early to be at some stage later than the primary visual cortex but, nonetheless, in posterior brain regions, whereas late equates to activation in the prefrontal cortex (Conci et al., 2004; Elliott et al., 2003).

\section{AUTHOR NOTE}

This research was supported by Deutsche Forschungsgemeinschaft Grant EL248/1 to M.A.E. The authors are indebted to Hermann Müller, James Townsend, Hans Geissler, Michael Kubovy, Peter van der Helm, and two anonymous reviewers for insightful comments and useful suggestions. The authors also express their thanks to Shanshan Chen, Doerthe Seifert, Goutami Shenvi, and Pilar Gamez-Moreno for their assistance in carrying out the experimental work. Correspondence concerning this article should be addressed to Z. Shi, Abteilung für Allgemeine und Experimentelle Psychologie, Department Psychologie, LudwigMaximilians-Universität, Leopoldstr. 13, D-80802 Munich, Germany (e-mail: strongway@psy.uni-muenchen.de).

\section{REFERENCES}

Arnoult, M. D. (1960). Prediction of perceptual responses from structural characteristics of the stimulus. Perceptual \& Motor Skills, 11, 261-268.

Becker, C., Elliott, M. A., \& Lachmann, T. (2005). Evidence for impaired visuoperceptual organisation in developmental dyslexics and its relation to temporal processes. Cognitive Neuropsychology, 22, 499-522.

BELL, R. A. (1970). Application note 115: Principles of cathode-ray tubes, phosphors, and high-speed oscillography. Colorado Springs: Hewlett Packard.

Box, G. E. P., \& Cox, D. R. (1964). An analysis of transformations. Journal of the Royal Statistical Society: Series B, 26, 211-252.

Box, G. E. P., \& Cox, D. R. (1982). An analysis of transformations revisited, rebutted. Journal of the American Statistical Association, 77, 209-210.

Castelo-Branco, M., Goebel, R., Neuenschwander, S., \& Singer, W. (2000). Neural synchrony correlates with surface segregation rules. Nature, 405, 685-689.

Chen, M., \& Chen, K. C. (1982). A transformational analysis of form recognition under plane isometries. Journal of Mathematical Psychology, 26, 237-251.

CHen, M., \& Chen, K. C. (1987). A group model of form recognition under plane similarity transformations. Journal of Mathematical Psychology, 30, 321-337.

Conci, M., Elliott, M. A., Müller, H. J., Wendt, J., \& Becker, C. (2004). The dynamics of operations in visual memory: A review and new evidence for oscillatory priming. Experimental Psychology, 51, 300-310.

CRICK, F., \& KoCH, C. (1995). Are we aware of neural activity in primary visual cortex? Nature, 375, 121-123.

Crick, F., \& КосH, C. (1998). Consciousness and neuroscience. Cerebral Cortex, 8, 97-107.

Donderi, D. C. (2006). Visual complexity: A review. Psychological Bulletin, 132, 73-97.

Donnelly, N., Humphreys, G. W., \& Riddoch, M. J. (1991). Parallel computation of primitive shape descriptions. Journal of Experimental Psychology: Human Perception \& Performance, 17, 561-570.

Dorfman, D. D., \& Alf, E., JR. (1969). Maximum-likelihood estimation of parameters of signal-detection theory and determination of confidence intervals: Rating-method data. Journal of Mathematical Psychology, 6, 487-496.

Eckhorn, R., Bauer, R., Jordan, W., Brosch, M., Kruse, W., MunK, M., \& Reiтвоеск, H. J. (1988). Coherent oscillations: A mechanism of feature linking in the visual cortex? Multiple electrode and correlation analyses in the cat. Biological Cybernetics, 60, 121-130.

Elliott, M. A., Becker, C., Boucart, M., \& Müller, H. J. (2000). Enhanced $\mathrm{GABA}_{\mathrm{A}}$ inhibition enhances synchrony coding in human perception. NeuroReport, 11, 3403-3407.

Elliott, M. A., ConcI, M., \& Müller, H. J. (2003). Prefrontal cortex and the generation of oscillatory visual persistence. Behavioral \& Brain Sciences, 26, 733-734.

Elliott, M. A., Giersch, A., \& Seifert, D. (2006). Some facilitatory effects of lorazepam on dynamic visual binding. Psychopharmacology, 184, 229-238.

Elliott, M. A., Herrmann, C. S., Mecklinger, A., \& Müller, H. J. (2000). The loci of oscillatory visual-object priming: A combined electroencephalographic and reaction-time study. International Journal of Psychophysiology, 38, 225-241.

Elliott, M. A., \& MüLler, H. J. (1998). Synchronous information presented in $40-\mathrm{Hz}$ flicker enhances visual feature binding. Psychological Science, 9, 277-283.

ElliotT, M. A., \& Müller, H. J. (2000). Evidence for 40-Hz oscillatory short-term visual memory revealed by human reaction-time measurements. Journal of Experimental Psychology: Learning, Memory, \& Cognition, 26, 703-718.

ElliotT, M. A., \& Müller, H. J. (2001). Effects of stimulus synchrony on mechanisms of perceptual organization. Visual Cognition, $\mathbf{8}$, 655-677.

Engel, A. K., Kreiter, A. K., König, P., \& Singer, W. (1991). Synchronization of oscillatory neuronal responses between striate and extrastriate visual cortical areas of the cat. Proceedings of the $\mathrm{Na}$ tional Academy of Sciences, 88, 6048-6052.

FAHLE, M., \& Koch, C. (1995). Spatial displacement, but not temporal asynchrony, destroys figural binding. Vision Research, 35, 491-494.

Feldman, J. (2000). Bias toward regular form in mental shape spaces. Journal of Experimental Psychology: Human Perception \& Performance, 26, 152-165. 
Freiwald, W. A., Kreiter, A. K., \& Singer, W. (1995). Stimulus dependent intercolumnar synchronization of single unit responses in cat area 17. NeuroReport, 6, 2348-2352.

Gail, A., Brinksmeyer, H. J., \& Eckhorn, R. (2000). Contour decouples gamma activity across texture representation in monkey striate cortex. Cerebral Cortex, 10, 840-850.

Garner, W. R. (1970). Good patterns have few alternatives. American Scientist, 58, 34-42.

GARNER, W. R. (1974). The processing of information and structure. Potomac, MD: Erlbaum.

GraY, C. M., KöNIG, P., Engel, A. K., \& Singer, W. (1989). Oscillatory responses in cat visual cortex exhibit columnar synchronization which reflects global stimulus properties. Nature, 338, 334-337.

Gur, M., Beylin, A., \& SNODderly, D. M. (1997). Response variability of neurons in primary visual cortex (V1) of alert monkeys. Journal of Neuroscience, 17, 2914-2920.

He, S., Cavanagh, P., \& Intriligator, J. (1996). Attentional resolution and the locus of visual awareness. Nature, 383, 334-337.

HUYNH, H., \& FELDT, L. S. (1976). Estimation of the Box correction for degrees of freedom from sample data in randomized block and splitplot designs. Journal of Educational Statistics, 1, 69-82.

KANDIL, F. I., \& FAHLE, M. (2001). Purely temporal figure-ground segregation. European Journal of Neuroscience, 13, 2004-2008.

KofFKA, K. (1922). Perception: An introduction to the Gestalt-Theorie. Psychological Bulletin, 19, 531-585.

KoFFKA, K. (1935). Principles of Gestalt psychology. New York: Harcourt, Brace.

KöHLER, W. (1947). Gestalt psychology: An introduction to new concepts in modern psychology. New York: Liveright.

LEE, S.-H., \& BLAKE, R. (1999). Visual form created solely from temporal structure. Science, 284, 1165-1168.

Leeuwenberg, E. L. J. (1968). Structural information of visual patterns: An efficient coding system in perception. The Hague: Mouton de Gruyter.

LivingSTONE, M. S. (1996). Oscillatory firing and interneuronal correlations in squirrel monkey striate cortex. Journal of Neurophysiology, 75, 2467-2485.

Mavrides, C. M., \& Brown, D. R. (1969). Discrimination and reproduction of patterns: Feature measures and constraint redundancy as predictors. Perception \& Psychophysics, 6, 276-280.

NeISSER, U. (1967). Cognitive psychology. New York: Appleton-CenturyCrofts.

Palmer, S. E. (1991). Goodness, gestalt, groups, and Garner: Local symmetry subgroups as a theory of figural goodness. In G. Lockhead \& J. Pomerantz (Eds.), The perception of structure: Essays in honor of Wendell R. Garner (pp. 23-40). Washington, DC: American Psychological Association.

Parton, A., Donnelly, N., \& Usher, M. (2001). The effects of temporal synchrony on the perceived organization of elements in spatially symmetric and asymmetric grids. Visual Cognition, 8, 637-654

Pomerantz, J. R., \& Pristach, E. A. (1989). Emergent features, attention, and perceptual glue in visual form perception. Journal of Experimental Psychology: Human Perception \& Performance, 15, 635-649.

Rager, G., \& Singer, W. (1998). The response of cat visual cortex to flicker stimuli of variable frequency. European Journal of Neuroscience, 10, 1856-1877.

Ts'o, D. Y., \& GiLBERT, C. D. (1988). The organization of chromatic and spatial interactions in the primate striate cortex. Journal of Neuroscience, 8, 1712-1727.

Ts'o, D. Y., GilberT, C. D., \& Wiesel, T. N. (1986). Relationships between horizontal interactions and functional architecture in cat striate cortex as revealed by cross-correlation analysis. Journal of Neuroscience, 6, 1160-1170.

Usher, M., \& Donnelly, N. (1998). Visual synchrony affects binding and segmentation in perception. Nature, 394, 179-182.
VAN Der Helm, P. A., \& Leeuwenberg, E. L. J. (1991). Accessibility: A criterion for regularity and hierarchy in visual pattern codes. Journal of Mathematical Psychology, 35, 151-213.

Wertheimer, M. (1912). Experimentelle Studien über das Sehen von Bewegung. Zeitschrift für Psychologie, 61, 161-265.

\section{NOTES}

1. Previous research (Elliott \& Müller, 1998, Experiment 3) has shown that a mislocated synchronous premask does not appear to attract focal attention. In addition, unpublished work from the Müller lab (H. Müller, personal communication, 2004) shows that neither endogenous nor exogenous target cues (cues presented independently of and in addition to the prime) influence the magnitude of priming. There is also evidence indicating that the priming effects are unaffected by eye movements. Previous research (Elliott \& Müller, 1998, Experiments 1 and 3) has shown that priming effects are invariant with respect to premask matrix presentation time. This suggests that even when ample time and opportunity to move the eyes are given, eye movements, if present, do not seem to influence priming. Furthermore, in three published EEG studies (Conci, Elliott, Müller, Wendt, \& Becker, 2004; Elliott, Conci, \& Müller, 2003; Elliott, Herrmann, Mecklinger, \& Müller, 2000), the priming effects still maintained the usual magnitude $(20-30 \mathrm{msec})$ when eye movement artifacts were rejected prior to analysis.

2. Each signal detection task had the same stimulus design as that in the main experiment, except that the final static target matrix was replaced by a matrix of premask inducers. Thus, the static matrix display acted as a mask. The participants were asked to indicate whether the flickering premask matrix contained a grouping frame (i.e., a frame containing a priming stimulus) or not. The participants made two rated responses (certain-present, uncertain-present, uncertain-absent, or certain-absent). The sensitivity parameter $A_{z}$ (a measure of sensitivity that is equivalent to the area under the ROC curve; see Dorfman \& Alf, 1969) was calculated, and a further statistical test based on an asymptotic distribution was conducted for each value of $A_{z}$ for each individual participant.

3. An experiment was conducted to examine variations in target size versus inducer length. In this experiment, inducer lengths were equivalent across three different matrices - that is, triangle, square, and hexagon. The results showed that target detection RTs to triangle matrices were significantly slower than those to hexagon matrices (mean of 725 vs. $568 \mathrm{msec}$ ). This was most likely caused by different target size, since the hexagon target was six times larger than the triangle. On the basis of this, an experimental design with targets of the same size was selected for the experiments reported in the main body of the text.

4. A pilot experiment was conducted to test the effects of local inducer line orientation on priming, in order to address design issues related to Experiments 2 and 3. Two different nine-element matrices (square and diamond) and three different inducers (cross, $45^{\circ}$-rotated cross, and circle) were examined, with premask matrix presentation frequency set to $40 \mathrm{~Hz}$ and intra- and interphase premask multifactorially varied relative to targetpresent or -absent conditions. The experiment was a within-subjects, full factorial design with 12 participants ( 3 of them male; mean age, 26.5 years). A repeated measures ANOVA conducted on the exponents of the means of log-transformed correct RT distributions revealed the expected target $\times$ prime interaction to be significant $[F(1,11)=18.484, p<.01]$, whereas the main effect and all interactions with inducer type were insignificant. This indicates that although priming effects remain specific to target trials, the magnitude of priming is relatively invariant with respect to the precise type of inducers employed.

(Manuscript received March 7, 2005; revision accepted for publication May 15, 2006.) 